Ružena Kozmová

ORCID: 0000-0003-3606-4391

Universität der hl. Cyril und Method in Trnava, Trnava

\author{
Lucia Miháliková \\ ORCID: 0000-0001-9159-9255 \\ Universität der hl. Cyril und Method in Trnava, Trnava
}

DOI: $10.19195 / 0435-5865.143 .15$

\title{
Polysemie des Verbs als Problem der Semantik-Pragmatik-Schnittstelle
}

\begin{abstract}
s
Das Ziel des Beitrages ist es, auf die kontextuelle Variabilität der verbalen Syntagmen hinzuweisen. Das Verb als ein Träger der quantitativen und qualitativen Valenz prädestiniert und gestaltet die syntaktische und semantische Satzperspektive, wobei seine prototypischen Eigenschaften projiziert werden. Die prototypische, grundlegende Valenz ist nach dem Typ des Verbs entweder zu erweitern oder zu reduzieren, was in einem betreffenden Satzkontext entweder die Obligatheit oder die Fakultativität der Verbergänzung signalisiert. Ein Beispiel für die Erweiterung der syntaktischen und semantischen Valenz sind auch die Geräuschverben. Diese primär monovalenten, nicht-direktionalen Verben werden unter Einfluss des Kontextes zu den mehrvalenten, direktionalen Verben.
\end{abstract}

Schlüsselwörter: Verbvalenz, Bedeutung, Polysemie, kontextuelle (konzeptuelle) Metapher

\section{Verb polysemy as a problem of semantics-pragmatics interface}

The aim of this paper is to highlight the contextual variability of verbal syntagmas. A verb, as a medium of quantitative and qualitative valency, predestinates or shapes the syntactic and semantic sentence perspective in which its prototype properties are reflected. The prototype, basic valency, may be either expanded or reduced, according to the type of the verb, which in a given context means either a binding or a non-binding verbal complement. An example of syntactic and semantic valency enlargement are also the verbs of sound. From these originally monovalent, non-directional words become, due to the context, bivalent or trivalent, directional verbs.

Keywords: verb valency, meaning, polysemy, contextual (conceptual) metaphor 
Ružena Kozmová, Universität der hl. Cyril und Method in Trnava, Philosophische Fakultät, Lehrstuhl für Germanistik, Nám. J. Herdu 2, 91701 Trnava, Slowakei, E-Mail: kruzena@yahoo. com

Lucia Miháliková, Universität der hl. Cyril und Method in Trnava, Philosophische Fakultät, Lehrstuhl für Germanistik, Nám. J. Herdu 2, 91701 Trnava, Slowakei, E-Mail: luci.mihalikova@, gmail.com

Received: 28.06.2017, accepted: 18.05.2018

\section{Einleitung}

In dem Beitrag wird auf die Frage der Bedeutungsverschiebung in Bezug auf die Valenzsyntagmen eingegangen, wobei die These postuliert wird, dass der Bedeutungswandel der Verben durch semantische, syntaktische, bzw. pragmatische Veränderungen bedingt ist.

Als empirische Basis dienen Korpora der deutschen Sprache und das parallele deutsch-slowakische Korpus der Valenzsyntagmen des Verblexems rauschen. Es geht um ein monovalentes Verb, das dank seiner semantischen Spezifizierung der kontextuellen Valenzerweiterung unterliegt, die zur Bedeutungsverschiebung in der Richtung Geräusch - Bewegung führen kann. In diesem Sinne wird hier geprüft, wie das Geräusch durch die Sprachmittel zum Ausdruck gebracht wird und unter welchen Bedingungen das Geräusch als Bewegung modifiziert werden kann.

Im Folgenden wird zuerst die Bedeutung bzw. der Bedeutungswandel im Allgemeinen behandelt, dann wird das Geräusch als eine Größe charakterisiert, und zwar als etwas Konstantes, das unter bestimmten Bedingungen Züge einer zielgerichteten Bewegung aufweist. Es wird von der physikalischen Regel ausgegangen, dass sich der Schall mit einer bestimmten Schnelligkeit in der Umgebung ausbreitet. Darauf aufbauend werden dann einzelne deutsche Belegbeispiele mit den slowakischen konfrontiert. Die konfrontative Ausrichtung des Beitrags weist auf die typologischen Spezifika im Zusammenhang mit der Valenzerweiterung im Deutschen und Slowakischen hin.

\section{Der Bedeutungswandel der Verben im Allgemeinen}

„Unter welchen Bedingungen verändert sich die Bedeutung eines Verbs?“, ist zunächst die Frage, die geklärt werden sollte. Laut Blank (1997: 133 ff., ähnlich auch Keller, Kirschbaum 2003) sind es drei Assoziationsprinzipien, die die Bedeutungsveränderung des Verbs bewirken können:

1. Similarität;

2. Kontiguität;

3. Kontrast. 
Aufgrund dieser Prinzipien unterscheidet Blank 12 Typen des Bedeutungswandels: a) Metapher, b) Metonymie, c) Ellipse, d) Volksetymologie, e) Bedeutungserweiterung, f) Bedeutungsverengung, g) Kohyponymische Übertragung, h) Antiphrasis, i) Auto-Antonymie, j) Auto-Konversion, k) Bedeutungsverbesserung und Bedeutungsverschlechterung, 1) Bedeutungsabschwächung und Bedeutungsverstärkung.

Die Frage nach dem möglichen semantischen Verfahren, das einen Bedeutungswandel verursacht, ist nicht neu. Es gibt unterschiedliche Klassifikationsversuche. Geht man jedoch von der Tatsache aus, dass die Verben für die Kommunikation am geeignetsten sind, scheint der logisch-rhetorische Gesichtspunkt eine gute Ausgangsbasis zu sein. So verfährt auch Ullmann (1967/72: 188 ff.), der folgende logisch-rhetorische Kategorien unterscheidet: Bedeutungsverengung, Bedeutungserweiterung, Bedeutungsübertragung. Keller/Kirschbaum (2003: 15) sind jedoch der Meinung, dass die Termini Bedeutungsverengung und Bedeutungserweiterung nicht unabhängig von „klassenlogischen Fragestellungen verwendet werden können“ und außerdem ,eine unzulässige Gleichsetzung von Bedeutung und Extension evozieren“. Dazu äußert sich auch Blank (1997: 192), der meint, dass „,diese Termini selbst eigentlich irreführend sind“. Dieser Meinung ist auch Bechmann (2013: 227):

Mit Bedeutungserweiterung ist [...] eine Erweiterung der Extension, des Bezeichnungsvermögens verbunden, während das Semem um mindestens ein distinktives Merkmal reduziert wird, der intensionale Gehalt nimmt ab. Umgekehrt kommt bei der Bedeutungsverengung mindestens ein Merkmal hinzu, dafür wird der Bezeichnungsrahmen reduziert [...].

Es zeigt sich dementsprechend, dass in diesen Fällen von Bedeutungsdifferenzierung bzw. Bedeutungsgeneralisierung zu sprechen wäre, weil, wie auch Bechmann annimmt, diese Begriffe rein qualitative Kategorien darstellen. Der Terminus Bedeutungsverengung ist eine rein quantitative Messgröße in Bezug auf Intension und Extension. Für Differenzierung und Generalisierung bietet es sich an, von Typen der Bedeutungsverschiebung zu sprechen. Dieser Meinung möchte ich mich anschließen, denn die Extension muss streng genommen nicht mit der Bedeutung, sondern vielmehr mit dem Bezeichnungsrahmen gleichgesetzt werden. Für die Zwecke des vorliegenden Beitrags bleibt im Weiteren der Bedeutungswandel wichtig, der durch Metaphorisierungsprozesse entsteht, wobei Metaphorisierung hier im weiteren Sinne verstanden wird, also nicht nur die Metapher, sondern auch die Metonymie mit einschließt. Die Interpretation metaphorischer und metonymischer Wortverwendung verläuft nach Bechmann (2013: 233) wie folgt: „Durch die Verregelung des neuen Sinns im Zuge der Lexikalisierung verliert das Wort seinen Assoziationscharakter und gewinnt einen Regelcharakter, der als lexikalische Bedeutung ohne Umwege erschlossen wird."1

Im Folgenden wird diskutiert, welchen Stellenwert Metapher und Metonymie für den verbalen Bedeutungswandel besitzen und unter welchen Bedingungen sie nachweisbar sind. Darauf bezieht sich die Frage, wodurch die Verwendung

\footnotetext{
${ }^{1}$ In Anlehnung an Keller/Kirschbaum (2003).
} 
eines semantischen Verfahrens bestimmt ist. Laut Bechmann (2013: 234) hängt die Möglichkeit der Anwendung semantischer Verfahren von drei wesentlichen Komponenten auf der Mikroebene des Sprechers ab:

1. Von der individuellen sprachlichen Kompetenz;

2. von der pragmatischen Eignung eines Wortes;

3. von der Verbreitung (oder Konventionalisierung) der sprachlichen Neuerung.

Für die Punkte 2, 3 gilt jedoch, dass sich nicht jedes Verb dazu eignet, in abweichender Weise verwendet zu werden. Umso mehr gilt dies bei Verbsyntagmen, da sowohl das Verb als auch das Substantiv ähnliche semantische Merkmale haben müssen. Es gilt dabei folgerichtig die Bedingung einer relativen Kompatibilität der beiden Bestandteile der Verbsyntagmen.

Nehmen wir als Beispiel das Verb fliegen und das Verbsyntagma durch die Prüfung geflogen (sein). Das Verb fliegen setzt mindestens Seme wie [belebt], [konkret] voraus, was im Falle des Substantivs Prüfung nicht erfüllt wird. Das Sem [konkret] wäre dann ausgeschlossen, weil das Verbsyntagma mit der Präposition durch weder durch die Maschine noch durch den Mähdrescher/Computer möglich ist. Die semantische Bedingung ist dann auch mit der Pragmatik des Wortes verbunden, denn weiter in diesem Sinne erwägend müssten wir uns die Frage stellen, ob das Sem [konkret] doch nicht anwendbar wäre, wenn es z.B. um das Verbsyntagma durch den Tunnel fliegen ginge. Die Bedingung in diesem Fall müsste dann das Sem [durchfahren möglich] erfüllen.

Metonymien resultieren aus konzeptuellen assoziativ bedingten Verschiebungen, sie bilden nach Dölling ${ }^{2}$ unterschiedliche Typen. Metonymische Interpretationen entstehen aus einer vom Kontext unterstützten systematischen Umformung einer wörtlichen Bedeutungsvariante des betreffenden Ausdrucks und befinden sich im selben „Sinnbereich“ (Bechmann 2013: 235). Metaphorische Interpretationen sind Bedeutungsverschiebungen, die zugleich eine Veränderung im vorausgesetzten Konzeptualisierungsraster einschließen, also „verschiedene Sinnbereiche" abdecken. Bei der Metonymie ist die Beziehung zur wörtlichen Bedeutung eine ontologische Relation zwischen Elementen derselben Domäne. Bei der Metapher wird hingegen Bedeutung aus einer Übertragung von einem Sinnbereich auf einen gänzlich anderen generiert.

\section{Verbale Metaphern}

Bedeutungswandel ist ein Phänomen, das nicht nur auf der Ebene des Nomens manifestiert wird, sondern oft bei den Verben der Fall ist, indem Veränderungen der Satzstrukturen bewirkt werden. Wenn man die These postuliert, dass der Metaphorisierungsprozess für die Kommunikation von Bedeutung ist, dann gilt es

${ }^{2}$ Vgl. Dölling (1999: 31 f.). 
für die Verben und seine Syntagmen umso mehr. In Bezug auf die Bildung von Metaphern bei Verbsyntagmen ist zweierlei wichtig: Zum einen gelten für die Bildung der Metapher die oben genannten Ausführungen, zum anderen sind es semantische Merkmale des nominalen Teils, die mit denen des Verbs kompatibel sein müssen, indem mindestens ein neues Sem des Substantivs mit denen des Verbs kompatibel ist. Es gilt folgerichtig, dass die Wandelphänomene bei Verben in der Richtung konkret - abstrakt vorgehen. Es stellt sich die Frage, ob die Valenz als Fähigkeit des Verbs Leerstellen zu eröffnen ,ein integraler Bestandteil der Verbbedeutung ist". ${ }^{3}$ Ich nehme an, dass eine solche Frage falsch gestellt wird. Es geht nicht darum, ob ,es daher richtig ist, semantischen Wandel über ein grammatisches Theorem beschreiben und erklären wollen". ${ }^{4}$ Die Frage ist also nicht, ob das Syntaktische dem Semantischen übergeordnet ist. Die semantischen wie die syntaktischen Parameter sind Vertreter der Seme der Verbbedeutung, wobei die Seme innerhalb der Verbbedeutung nicht nur horizontal, sondern auch vertikal geordnet sind, indem sie in einer koordinativen wie auch subordinativen Beziehung zueinander stehen. Die Ergänzungsbedürftigkeit des Verbs ist immer auf einen bestimmten Aktanten angewiesen, um damit als Valenzsyntagma zu funktionieren. Daher ist die Valenz ein semantisch-syntaktisches Phänomen, das die Semantik und die Syntax als ein untrennbares Ganzes darstellt, und auf diese Verbundenheit macht die Analyse der Valenzsyntagmen des Verbs rauschen deutlich.

\subsection{Die Grundvalenz des Verbs rauschen}

Die Grundvalenz des Verbs rauschen bilden die monovalenten, imperfektiven ${ }^{5}$ Syntagmen. Die semantische Konzeptualisierung bezieht sich bei diesem Verb auf die Art und die Intensität des Geräusches. Das Geräusch ist zu charakterisieren als etwas, das akustisch mehr oder weniger stark wahrgenommen wird (und das ohne bewusste Absicht durch etwas in Bewegung Befindliches oder Gesetztes entstanden ist). ${ }^{6}$ Wie die Definition selbst andeutet, sind das Geräusch und die Bewegung ursächlich verknüpft. Die Kausalität dieser zwei Größen besteht darin, dass das Geräusch aus der physikalischen Sicht als eine mechanische Schwingung einer materiellen Umgebung ${ }^{7}$ zu bezeichnen ist, die man hören kann, wobei damit die schwingende Bewegung der Luftteilchen oder einer anderen Umgebung, z.B. des Wassers, gemeint wird. Die Semantik des Verbs rauschen, die von der Semantik des Substantivs das Geräusch ausgeht, beschreibt primär das Geräusch und die

3 Vgl. Bechmann (2013: 271).

${ }^{4}$ Vgl. Bechmann (ebd.).

${ }^{5}$ Die imperfektiven monovalenten Verben im Slowakischen decken sich meistens mit den monovalenten Tätigkeitsverben im Deutschen.

${ }^{6} \mathrm{http}: / /$ www.duden.de/rechtschreibung/Geraeusch_Laut_Ton_Brummen.

${ }^{7}$ http://slovniky.juls.savba.sk/ Krátky slovník slovenského jazyka 4 (2003), Slovník slovenského jazyka (1959-1968). 
Bewegung der Naturphänomene. Die Geräuschintensität bestimmt die konkrete Substantivergänzung, mit der sich das Verb rauschen in einem Kontext verbindet. Der prototypische Gebrauch des monovalenten Verbs rauschen signalisiert ein natürliches Geräusch, das z.B. durch die Bewegung der Luft (1), durch die Bewegung der Wassertropfen (2) oder durch die Bewegung eines kleineren Wasserlaufes (3) entsteht. Die Intensität des Geräusches, die durch Subjektergänzungen wie die Blätter, der Regen und der Bach erzeugt wird, ist sanft, sie ist dem Geräusch von dem sich langsam bewegenden Wasser oder von den sich im Wind leise bewegenden Blättern, anzugleichen. Das Verb rauschen ist somit ins Slowakische mittels der Äquivalente šušstat', šumiet', žblnkat', žblnkotat', zurčat' (1a), (2a), (3a) zu übersetzen. Ein weniger intensives Geräusch kann im Slowakischen durch die verbalen Suffixe ausgedrückt werden. Ein Beispiel dafür ist die diminutive Entsprechung žblnkotat'.

Die polysemische Antonymie ${ }^{8}$ des Verbs rauschen, in Bezug auf die unterschiedliche Abstufung der Geräuschintensität, entspricht seiner Grundsemantik. Die gegensätzliche Bedeutung des Verbs rauschen, also diese, in der die Geräuschintensität stärker ist, manifestiert sich dann, wenn es z.B. um die Bewegung eines größeren Wasserlaufes (5) oder um die Bewegung einer massiven Wasserschicht (6) geht. Ein dumpfes Geräusch, das der Fluss oder das Meer erzeugen, ist ein Begleitphänomen einer größeren und schnelleren Wasserströmung. Die slowakische Entsprechung ist in den betreffenden Syntagmen das Verb hučat' (4a), (5a).

(1) Der Wind pfeift, die Blätter rauschen.

(1a) Vietor píska, listy šuštia.

(2) In den Bäumen um sie herum rauschte der Regen.

(2a) V stromoch šušt’al dážd'.

(3) Der Weg stieg nicht mehr, und der Bach rauschte jetzt neben ihnen.

(3a) Cesta prestala stúpat' a potok šumel/žblnkal/žblnkotal/zurčal vedl'a nich.

(4) Hinter ihnen rauschte in der Tiefe der Fluss.

(4a) Kdesi v híbke za nimi hučala rieka.

(5) Verzweifelt blickte sie hinunter, wo das Meer rauschte und schäumte.

(5a) Zúfalo sa zahl'adela dolu, kde hučalo spenené more.

Die prototypische Semantik des Verbs rauschen, in Gestalt des Geräusches der Naturphänomene, wird zur kognitiven Basis für die Entstehung weiterer, abgeleiteter verb-nominaler Syntagmen. Die kognitiven Mechanismen, basierend auf dem Ähnlichkeitsverhältnis, kommen dann zum Vorschein, wenn es sich um die metaphorische Übertragung der Eigenschaften des einen Konzeptes auf das andere Konzept handelt. So können nicht nur die Blätter rauschen, sondern auch ein gewisses Material (6). Der Satz (6) benennt das Knistergeräusch eines Klei-

${ }^{8}$ In der slowakischen Linguistik wird die polysemische Antonymie mit dem Begriff Enantiosemie bezeichnet. 
derstoffes, konkret der Roben (oft aus Seide), das aufgrund der Bewegung, resp. der Reibung der Materialschichten entsteht. Das Knistergeräusch kann auch ein Gerät (7) erzeugen. Als eine Bewegung ist hier die physikalische Ausbreitung eines bestimmten Geräuschniveaus zu beschreiben. In dem Beispiel (7) rauschen Fernseher wegen der Beeinträchtigung der Signale.

(6) Im prachtvollen Marmorsaal rauschen dann die Roben, auch Touristen können Eintrittskarten für den Ball erwerben.

(6a) V nádhernej mramorovej sále šuštia róby, aj turisti môžu získat' vstupenky na ples.

(7) Viele neuere Fernseher brummen oder rauschen erstaunlicherweise, selbst wenn die Lautstärke auf den kleinstmöglichsten Wert gestellt wurde.

(7a) Mnoho nových televízorov hučí alebo šuští napodiv aj vtedy, ked’ je hlasitost' nastavená na najmenšiu možnú intenzitu.

Die Semantik der Verben donnern und knattern ist präsent in den Syntagmen (8) und (9), und dies setzt voraus, dass die Geräuschintensität im Vergleich zu den Syntagmen (6), (7) stärker ist. Wenn die Motoren rauschen, so wird damit ihr lauter technischer Betrieb gemeint. Ein donnerndes Geräusch kann metaphorisch auch ein Fest erzeugen, wenn es um ein Ereignis geht, an dem Menschen bei lauter Musik teilnehmen. Das Geräusch entsteht im Falle eines Motors aufgrund einer technischen Bewegung. Der Satz (9) bezieht sich auf die verbale und die nonverbale Ausbreitung des Geräusches.

(8) Der Kran surrt, die Motoren rauschen, die Bauarbeiter schicken Rufe hin und her, der Kranführer empfängt Befehle über Funk.

(8a) Žeriav vrčí, motory hučia/rachotia, robotníci kričia sem a tam, žeriavnik prijíma príkazy cez vysielačku.

(9) Draußen rauschte der Regen, drinnen ein Fest.

(9a) Vonku burácal dážd', vnútri burácala oslava.

Die Geräuschausbreitung selbst ist aus der physikalischen Sicht eine Art der Bewegung. In den Syntagmen (1)-(5) geht es um ein natürliches Geräusch. Das technische Geräusch ist in den Syntagmen (7)-(9) zu betrachten, und in Satz (6) ist das Geräusch ebenso künstlich geschaffen.

\subsection{Die Grundvalenzerweiterung des Verbs rauschen}

Die Grundvalenz des Verbs kann in einem bestimmten sprachlichen Kontext quantitativen und qualitativen Veränderungen unterliegen. Die quantitativen Veränderungen hängen mit der Veränderung der syntaktischen Struktur des Verbs zusammen, die in der Regel die Veränderung der qualitativen Struktur des Verbs bedingt (und umgekehrt). Die Veränderung der Grundvalenz des Verbs ist laut des konstruktionsgrammatischen Ansatzes von Welke (2015: 3) ein Ausdruck der innovativen Kommunikationsabsicht der Sprachproduzenten: „Es kommt vor, dass Sprecher neue 
Instantiierungen von Konstruktionen ausprobieren, indem sie Verben an Konstruktion anpassen (coercion), in die sie zuvor noch nicht fusioniert worden sind. Diese coercion führt zu einer Ad-hoc-Bedeutungsänderung des betreffenden Verbs.“ Die Bedeutungsveränderung des Verbs ist aus der Sicht der quantitativen Valenz durch die konzeptuelle bzw. kontextuelle Anpassung des Verbs ,,an geringerstellige oder höherstellige Konstruktionen" (ebd.: 19) beeinflusst, die die Fakultativität oder die Obligatheit der Verbergänzung voraussetzen. Bei der Valenzerweiterung der Geräuschverben geht es um die Obligatheit der zweiten bzw. dritten Verbergänzung. Das heißt, dass die Geräuschverben, die im Lexikon als monovalente Verben verzeichnet sind, unter dem Einfluss des Kontextes einen bivalenten oder trivalenten Charakter erwerben können. Eine neue syntaktische Charakteristik hängt mit einer qualitativ neuen semantischen Spezifizierung der Geräuschverben zusammen, die sich auf ihre Bewegungs- und Direktionalinterpretation ${ }^{9}$ bezieht. Die direktionalen Syntagmen mit den Geräuschverben stellen eher die Valenzperipherie dar, weil sie ein Resultat der individuellen, der kreativen Arbeit mit der Sprache sind. Die Kreativität äußert sich vor allem ,,auf der kombinatorischen Ebene, in der Fähigkeit, die Zusammenhänge zwischen den Phänomenen zu finden, die Grundzüge aus ihnen auszuwählen und sie in das neue Ganze zu kombinieren“" (Krupa 1990: 30). ${ }^{10}$ Die Rolle der Metapher in dem sprachlichen Prozess betonen u.a. auch Connor/Kogan (in Krupa: ebd.). Die Autoren fassen die Metapher als „die Fähigkeit, die konzeptuellen Verbindungen zwischen den Objekten oder Ereignissen aus den disparaten Ebenen zu finden, störend die Grenzen der geläufigen Kategorien“, auf. ${ }^{11}$ Die Metaphorisierung bezieht sich im Bereich der Verbvalenz auf zweierlei:

a) Unter der Disparatheit ist die semantische Inkompatibilität der Verbseme mit denen des Substantivs zu verstehen.

b) Die Störung der Grenzen der geläufigen Kategorien durch die Metapher impliziert die kontextuelle Veränderung der Grundvalenz des Verbs. Es geht um ein gewisses kognitiv gesteuertes Experiment, das sich nicht nur auf der lexikalischen, sondern auch auf der grammatischen Ebene manifestieren kann. Die Bezeichnung Experiment wird deswegen verwendet, weil die kontextuell akzeptable Extension der syntaktischen und der semantischen Verbvalenz mit der Frage nach der Sprachrezeption zusammenhängt, und dies bedeutet, dass der Blick auf die kreative Seite der Sprache individuell ist. ${ }^{12}$

${ }^{9}$ Vgl. dazu Engelberg (2009), Eroms (2012), Welke (2005, 2015).

10 Übersetzt von L.M.

11 Übersetzt von L.M.

12 Krupa (1990: 29): „Die Bildungskraft ist immer ein individueller Akt, ein Werk des Einzelnen im Gegensatz zur Gesellschaft, die die Innovation konventionalisieren und sanktionieren kann, sodass sie allgemein verständlich und akzeptabel für alle Sprachbenutzer wird. Natürlich, nicht jede Metapher muss allgemein akzeptabel sein - und die Gemeinschaft, obwohl sie selbst keine Innovationen gestaltet, entscheidet darüber, ob sie die Ausdrucksmittel der Sprache erweitern, oder ob sie abgelehnt werden.“(Übersetzt von L.M.) 
Die kognitive Ausgangsbasis bildet im Falle des Verbs rauschen das Geräusch und die Bewegung der Naturphänomene, deren Kausalität (metaphorisch) auch in den direktionalen Syntagmen enthalten sein kann. Es zeigt sich, dass die Valenzerweiterung der Geräuschverben durch die direktionalen Konstruktionen, bzw. Ergänzungen, mit der Aktionalität des verbalen Geschehens zusammenhängen kann. Die resultative Aktionsart ist somit im Falle des Verbs rauschen analytisch ausgedrückt, und dies mittels der spezifischen direktionalen Konstruktionen, in denen das Verb kontextuell verwendet wird. Daraus ergibt sich, dass zur Realisierung der resultativen Aktionsart bei einer gewissen Verbklasse die spezifischen Typen der syntaktischen Konstruktionen vorausgesetzt werden. In diesem Zusammenhang gerät nicht nur die Valenz der Verben selbst, sondern auch die Valenz der kompositionellen Aktionsarten in den Vordergrund. Daher ist zu Recht anzunehmen, dass nicht nur die Verben, sondern auch die Aktionsarten als resultative Konstruktionen ergänzungsbedürftig sind (Kozmová 2013: 116).

Die Analyse der erweiterten Valenz des Verbs rauschen konzentriert sich auf die kontextuelle Polysemie dieses Verbs, die ein Resultat der Metaphorisierung ist. Die Direktionalität des Verbs rauschen entsteht mithilfe der analytischen Konstruktionen mit den direktionalen Verbzusätzen, den direktionalen Adverbien ${ }^{13}$ und den direktionalen Präpositionen. Sämtliche Arten dieser analytischen direktionalen Konstruktionen sind in Korpora zahlreich vertreten, aus Platzgründen wird nur ein Teil davon exemplifiziert. Dabei werden die zentralen und peripheren Phänomene sowie die semantisch-pragmatische Vielfalt in Bezug auf die Repräsentanz der substantivischen Ergänzungen berücksichtigt. Die Gegenüberstellung der deutschen Sätze mit deren slowakischen Äquivalenten deckt die typologischen Unterschiede zwischen den beiden Sprachen auf, zu denen u.a. der analytische Charakter des Deutschen und der synthetische Charakter des Slowakischen gehören.

\subsubsection{Analytische Konstruktionen mit den ausgewählten direktionalen Verbzusätzen}

Der Ausdruck „Verbzusatz” charakterisiert einen trennbaren Teil „unfest zusammengesetzter Verben, die bei Satzkonstruktionen mit Zweistellung des Verbs als klammerschließendes Element auftreten“ (Bußman 1990: 831, vgl. dazu auch Glück 2005: 721). Eine Analogie zu diesem Ausdruck ist der Begriff „Parti-

${ }^{13}$ Für die verbale Komposition sind laut Fleischer (1995: 292) die Adverbien von großem Nutzen bzw. „die Komposition aus Adverb und Verb ist stärker entwickelt“, wobei „,besonders häufig Richtungsadverbien als Erstglieder auftreten“. Fleischer (ebd.) merkt zugleich an, dass „die Kombination der Richtungsadverbien mit Verben der Fortbewegung kaum Beschränkungen unterliegt“. Besonders wortbildungsaktiv sind laut Fleischer (ebd.: 301) die Richtungsadverbien hin- und herund ,die entsprechenden Zusammensetzungen mit Präpositionaladverbien“. Die Dominanz der verbalen Zusammensetzungen mit Adverbien her- und hin-, die der lokalen Differenzierung dienen, betont auch Lohde (2006: 222 f.): „Bei der Darstellung der Räumlichkeit kann auf eine große Modellvielfalt zurückgegriffen werden; man bevorzugt jedoch Komposita mit hin- und her-.“ 
kel(verb)", auch Partikelkompositum genannt. Laut Bußman (1990: 562) stehen neben den sogenannten Partikeln in der Regel homonyme Präpositionen oder Adverbien.

Aufgrund dieser These werden bei dem Verb rauschen zwei Typen der direktionalen Verbzusätze unterschieden: präpositionale und adverbiale. ${ }^{14}$ Der semantische und pragmatische Status der exemplifizierten direktionalen Verbzusätze wurde durch das Duden-Wörterbuch und das DWDS-Wörterbuch überprüft.

\subsubsection{Der präpositionale Verbzusatz $a b$ -}

Die Verbindbarkeit des Verbs rauschen mit der Präposition $a b$ in der Funktion des Verbzusatzes ist sowohl im Duden- als auch im DWDS-Wörterbuch belegt. Das Duden-Wörterbuch verweist auf den umgangssprachlichen Gebrauch dieses Prädikats in den Bedeutungen ,a) sich rasch (mit Auto, Motorrad o. Ä.) entfernen, b) sich auffällig entfernen“15, womit die Bedeutung ,rauschend davongehen“ ${ }^{\prime 16}$ mit der Konnotation „salopp“ im DWDS-Korpus kongruent ist. Ein Beleg für die auffällige und schnelle Bewegung eines Fahrzeuges ist der Satz (10). Die Bedeutung des Verbs rauschen in der Verbindung mit dem präpositionalen Verbzusatz $a b$ - ist in dem betreffenden Kontext ins Slowakische mittels der perfektiven Äquivalente odist'odfrčat'(10a) zu interpretieren.

(10) Nach der Pressekonferenz im „Hilton“ rauschte Bundestrainer Jürgen Klinsmann (41) mit Oliver Bierhoff, Jogi Löw und Andy Köpke in einer schwarzen Mercedes-Limousine Richtung Düsseldorfer Zentrum ab.

(10a) Po tlačovej konferencii v hoteli "Hilton" odišiel/odfrčal spolkový tréner (41) s Oliverom Bierhoffom, Jogim Löwom a Andym Köpkem v čiernej limuzíne značky Mercedes do düsseldorfského centra.

\subsubsection{Der präpositionale Verbzusatz zu-}

Ein charakteristisches konstruktionelles Element des analytischen Prädikats zurauschen ${ }^{17}$ ist die direktionale Präpositionalergänzung mit der Präposition auf. Die Konstruktionen dieses Typs können beispielsweise die direktionale Bewegung eines Naturphänomens (11) oder die Metaphorisierung der direktionalen Bewegung mit

${ }^{14}$ Im DWDS-Korpus sind bei dem Verb rauschen insgesamt 19 direktionale Verbzusätze zu finden, davon tragen 6 einen präpositionalen und 13 einen adverbialen Charakter; für Komplexität ist ihr quantitatives Vorkommen $\mathrm{zu}$ vergleichen: vorbeirauscht (1076), heranrauscht (180), aufrauscht (179), vorüberrauscht (134), durchrauscht (124), abrauscht (120), anrauschte (86), hinwegrauscht (54), hereinrauscht (51), zurauscht (51), niederrauscht (36), herunterrauscht (31), hineinrauscht (17), davonrauscht (17), zusammenrauschen (16), dahinrauscht (16), hinunterrauscht (14), hinabrauschte (11), entlangrauscht (8).

$15 \mathrm{http} / / / \mathrm{www}$.duden.de/rechtschreibung/abrauschen

16 https://www.dwds.de/wb/ab-

17 Der präpositionale Verbzusatz $z u$ - drückt die Richtung auf jmdn., etw. aus: https://www. dwds.de/wb/zu-. 
dem semantischen Merkmal einer Person (12) ausdrücken. In dem Satz (11) ist die schnelle Bewegung eines Naturphänomens vorauszusetzen, die im Slowakischen das perfektive Prädikat privalit' repräsentiert. Im Satz (12) geht es im Gegenteil um einen schnellen Verlauf einer Situation, ausgedrückt durch das slowakische imperfektive Äquivalent rútit'sa.

(11) Die Verwerfung im Meeresboden löste einen Tsunami aus, der innerhalb von Minuten mit bis zu 30 Meter hohen Wellen auf die Küste Sumatras zurauschte.

(11a) Zlom v morskom dne spôsobilo/vyvolalo tsunami, ktoré v priebehu minút dovalilo na pobrežie Sumatry až 30 metrov vysoké vlny.

(12) Wir rauschen auf eine schwere Wirtschaftskrise zu, aber niemand hat das Steuer in der Hand.

(12a) Rútime sa do t’ažkej hospodárskej krízy, ale nikto nedrží kormidlo (pevne) v rukách.

\subsubsection{Der adverbiale Verbzusatz vorbei-}

Die umgangssprachliche Bedeutung ,sich rauschend vorbeibewegen“18 ist mit dem Prädikat vorbeirauschen verbunden. Diese verbale Situation lässt eine Bewegung erkennen, ,die auf eine Person, Sache zu, neben dieser her und wieder von ihr weg verläuft" ${ }^{19}$ In dem Belegbeispiel (13) geht es um eine direktionale Bewegung eines Fahrzeuges, des Fernzuges, der sich schnell und rauschend vorbeibewegt. Ein solches Verbalereignis ist ins Slowakische mittels des perfektiven Äquivalents prehrkotat' (13a) zu übersetzen.

(13) Die Fernzüge, die ab und an mal vorbeirauschen, hören sie gar nicht mehr.

(13a) Dial'kové vlaky, ktoré tu občas prehrkocú, už vôbec nepočujú.

\subsubsection{Der adverbiale Verbzusatz zusammen-}

„Die Richtung von oben nach unten, die das Zubodensinken, Umfallen einer Person bezeichnet" ${ }^{20}$ ist in dem Prädikat zusammenrauschen (14) mit enthalten. Diesem Verb entspricht nach dem Duden-Wörterbuch das umgangssprachliche, figurative Prädikat zusammenrasseln ${ }^{21}$ dessen kontextuelle Konnotationen desgleichen für das Verb zusammenrauschen gelten. Der betreffende verbale Sachverhalt, bezogen auf ein Aufeinanderprallen von zwei Personen, ist im Slowakischen durch das perfektive Äquivalent zrazit'sa (14a) vertreten.

$18 \mathrm{http} / /$ www.duden.de/rechtschreibung/vorbeirauschen, https://www.dwds.de/wb/vorbeirauschen.

19 https://www.dwds.de/wb/vorbei-.

20 Vgl. https://www.dwds.de/wb/zusammen-.

${ }^{21} \mathrm{Vgl}$. http://www.duden.de/woerterbuch/deutsch-englisch/zusammenrauschen. 
(14) Kurz vor der Pause rauschte Meichelbeck bei einem Angriff der Bayern mit dem Kollegen Tommy Bechmann zusammen.

(14a) Krátko pred pauzou sa zrazil Meichelbeck pri útoku mužstva Bayern s kolegom Tommy Bechmannom.

\subsubsection{Analytische Konstruktionen mit den ausgewählten direktionalen Adverbien}

Die analysierten Syntagmen des Verbs rauschen mit den direktionalen Adverbien entstammen dem DWDS-Korpus, wo die folgende quantitative Übersicht der Verbindbarkeit des Verbs rauschen mit dieser Art der direktionalen Mittel zu finden ist: vorbei (92), unten (65), hinein (36), ran (35), herab (30), runter (29), heran (28), hinab (27), hinunter (25), herauf (15), hindurch (15), entlang (12), rauf (9).

\subsubsection{Das direktionale Adverb herab}

Das Verb rauschen kann in Verbindung mit dem Lokaladverb herab die Direktionalität eines Naturphänomens benennen. In den Belegbeispielen ist die direktionale Bewegung dem Regen zugeschrieben, der im wörtlichen Sinne von oben nach unten fällt, und dies signalisiert in diesem Fall eben das Adverb herab in dem deutschen Satz (15), das in der gehobenen Sprache für herunter gebraucht wird. ${ }^{22}$ In dem angeführten Beispiel beteiligt sich das Adverb herab an der Bewegungsinterpretation des Verbs rauschen. Jedoch bestimmt die Art und Weise des Ereignisses das Adverb endlich an der Bildung des analytischen perfektiven ${ }^{23}$ Prädikats, das ins Slowakische synthetisch durch das präfigierte perfektive Äquivalent spadnút (15a) wiedergegeben wird. In dem slowakischen Äquivalentsatz verändert sich aufgrund dieses Bewegungsverbs der formale Ausdruck des Adverbs herab, das in dem deutschen Satz explizit realisiert ist, während bei dessen slowakischem Äquivalent von dem impliziten Ausdruck des betreffenden verbalen Ereignisses zu sprechen ist. Die wörtliche Äquivalenz ist auch in Satz (16) ausgeschlossen. Das Äquivalent padat' dolu (16b) weist zwar auf die reale Erklärung des betreffenden verbalen Geschehens hin, wie auch auf das Merkmal der Bewegung, jedoch sind aus der Sicht der komplexen Satzsemantik eher korrekt die metaphorischen imperfektiven Äquivalente neutíchat'/neustávat' (16a).

(15) Endlich rauschte der Regen auf die dürr gewordene Stadt herab.

(15a) Na vyschnuté mesto spadli konečne výdatné zrážky.

(16) Der Regen rauschte ohne Unterlass herab.

(16a) Dážd’ neutíchal/neustával.

(16b) *Dážd’ padal dolu.

$22 \mathrm{http} / / /$ www.duden.de/rechtschreibung/herab.

${ }^{23}$ Der Verbalkategorie Aspekt in den slawischen Sprachen entspricht im Deutschen die Kategorisierung in prozessuale und resultative Prädikate, die meistens einen analytischen Charakter haben. Das Adverb bzw. Wörter, die einen adverbialen Zug aufweisen, sind die relevantesten Bestandteile des Satzes. Je nach der semantischen Charakteristik des Adverbs unterscheidet man durative/ nicht resultative oder resultative Prädikate. 


\subsubsection{Das direktionale Adverb hinab}

Ein Gegenbeispiel zur expliziten Nicht-Realisierung des Richtungsadverbs dolu im Slowakischen ist das Syntagma (17a). Hier ist das Richtungsadverb dolu (hin$a b)$ eher obligatorisch, weil es den semantisch-pragmatischen Kontext des Satzes abgrenzt. Wenn man sagt, dass 3210 Kubikmeter Wasser pro Sekunde den Fluss hinabrauschten, meint man somit die Menge des Wassers, die sich den Fluss hinabbewegte. Das direktionale Adverb hinab ist eine gehobene Konnotation des direktionalen Adverbs hinunter. ${ }^{24}$

(17) Am Morgen rauschten nach Behördenangaben 3210 Kubikmeter Wasser pro Sekunde den Fluss hinab - normal sind 150.

(17a) Ráno pretieklo podl'a štatistických údajov dolu riekou za sekundu až 3210 metrov kubických vody, za normálnych okolností je to 150 .

\subsubsection{Analytische Konstruktionen mit den ausgewählten direktionalen Präpositionen}

Die direktionalen Präpositionen, ähnlich wie die direktionalen Adverbien, gehören im Deutschen zu denjenigen Mitteln, die bei der analytischen Bildung der Aktionsart anzuwenden sind. In der Verbindung mit einer konkreten substantivischen Ergänzung tragen sie zu einer vielfältigen semantischen Variation des Verbs rauschen bei.

\subsubsection{Die direktionale Präposition aus}

Die dativische Präposition, die sich mit der Direktionalität des Verbs rauschen verknüpft, ist die Präposition aus. Das typische Merkmal der Syntagmen, in denen dieser Direktionalausdruck vorkommt, ist in der Regel das auffällige Verhalten einer Person, das von einer schnellen Bewegung und metaphorisch auch von einem Geräuschphänomen begleitet ist. Die semantische Variabilität, in Gestalt der kontextuellen Anpassung der Verbbedeutung, reflektiert die Äquivalenz im Slowakischen. Dem analytischen Prädikat aus etwas rauschen (18), (19), (20) entspricht im Slowakischen das synthetische Prädikat mit dem Präfix vy-(18a), (19a), (19b), (20a), das die Perfektivität des verbalen Geschehens signalisiert.

(18) Er rauschte wütend aus dem Zimmer.

(18a) Rozzúrene vybehol z izby.

(19) Er rauschte mit überhöhter Geschwindigkeit aus der Kurve.

(19a) Prekročenou rýchlost’ou vybehol/vyletel zo zatáčky/vyrútil sa zo zatáčky.

(19b) Vyletel zo zatáčky prekročiac povolenú rýchlost’.

(20) Erhobenen Hauptes rauschte sie aus dem Saal

(20a) So vztýčenou hlavou vyplávala zo sály.

${ }^{24} \mathrm{http} / / /$ www.duden.de/rechtschreibung/hinab. 


\subsubsection{Die direktionale Präposition durch}

Der am meisten vertretene Direktionalausdruck ist die akkusativische Präposition durch. Die Subjektergänzungen, die sich mit dieser Präposition kontextuell verbinden, haben eine mannigfaltige semantische Klassifizierung.

Die erste Gruppe bilden die Syntagmen, die die direktionale Bewegung eines Fahrzeuges (21) oder eines Gegenstandes (22) benennen, wobei das Geräusch ein Begleitphänomen dieser Bewegung ist. Das Prädikat hrkotat' in dem Beispiel (21a) bezeichnet eine imperfektive Aktionsart, das präfigierte Prädikat preletiet' in dem Beispiel (22a) bezeichnet im Gegenteil eine perfektive, resultative Aktionsart. Die Gegenüberstellung der Sätze (22) und (22a) zeigt eine unterschiedliche morphologische Realisierung der zweiten Verbergänzung. Die analytische akkusativische Struktur rauschen durch den Korb ist im Slowakischen synthetisch realisiert, und dies mittels des Instrumentals preletiet' košom. Es geht um den Umstandsinstrumental. Der Instrumental des Ortes „drückt die Umgebung aus, wodurch die Handlung betroffen wird und kommt bei den Verben mit dem direktionalen Element vor" (Dvonč et al. 1966: 185).

(21) Straßenbahnen rauschen durch die Ludwigstraße.

(21a) Električky hrkocú po Ludwigovej ulici.

(22) Ohne Ringberührung rauschte der Ball durch den Korb.

(22a) Lopta preletela košom, bez toho, aby sa dotkla kruhu.

Die zweite Gruppe stellen die metaphorischen Syntagmen dar, in denen es sich eher um eine kreative Kommunikationsabsicht als um eine reale direktionale Bewegung des Subjektes handelt. Wenn die Gedanken (23) jmdm. sofort durch den Kopf rauschen, ist somit gemeint, dass die Gedanken jmdm. sofort durch den Kopffliegen. Dagegen kann der Student (24) durch etwas rauschen, speziell durchs Examen, wenn er die Prüfung nicht bestanden hat, er hat also die in der Prüfung geforderte Leistung nicht in ausreichender Form erbracht. „Das Moment des Fliegens" macht sich metaphorisch auch in dem wirtschaftlichen Bereich bemerkbar, insbesondere dann, wenn die Milliarden (25) durch den Kamin gerauscht sind und jemand so einen Geldverlust erleidet. Die perfektive, resultative Aktionsart impliziert in den Sätzen (24), (25) das sein-Passiv.

(23) Sofort rauschen besorgte Gedanken durch meinen Vater-Kopf.

(23a) Ustarostené myšlienky vyšumeli/vyleteli ihned' z mojej otcovskej hlavy.

(23b) Rýchlo som sa zbavil t’aživých myšlienok/vyšumeli mi z hlavy.

(24) Der Student, der durchs Examen gerauscht ist, telegrafiert seinem Bruder: „Nicht bestanden - stop - bereite Vater vor - stop!” Der Bruder telegrafiert zurück: „Vater vorbereitet - stop - bereite Dich vor - stop!”

(24a) Študent, ktorý vyletel zo skúšky (prepadol), telegrafuje svojmu bratovi: "Nespravil som - stop - priprav otca - stop!” Brat odpíše spät': “Otec pripravenýstop - priprav sa Ty - stop!" 
(25) Im Zusammenhang mit der neuen Ökonomie sind innerhalb von 18 Monaten auf dem deutschen Kapitalmarkt 600 Milliarden durch den Kamin gerauscht.

(25a) V súvislosti s novou ekonomikou vyletelo v priebehu 18 mesiacov na nemeckej burze 600 miliárd hore komínom.

$\mathrm{Zu}$ der dritten Gruppe gehören die Syntagmen, in denen sich das Geräusch durch einen Raum ausbreitet (26).

(26) Donnernder Applaus rauschte durch den Saal.

(26a) Búrlivý potlesk zaznel v sále.

Ein spezifisches Syntagma mit der Präposition durch stellt der Satz (27) dar. Der Agens der verbalen Situation ist ein Naturphänomen, der Wind, der sich mit den Blättern leise bewegt, und die Blätter erzeugen dabei ein sanftes Geräusch, was im Slowakischen durch das Äquivalent ševelit' (27a) repräsentiert ist.

(27) Der Wind rauscht durch die Blätter.

(27a) Vietor ševelí lístím.

\subsubsection{Die direktionale Präposition gegen}

Der Einfluss einer Naturkraft, die eine Bewegung und ein Geräusch durch das Subjekt bewirkt, prägt sich in dem Beispiel (28) aus, wo sich das Verb rauschen mit der Direktionalpräposition gegen verbindet. Das Verb rauschen ist in dem betreffenden Kontext mittels des imperfektiven Prädikats narážat' (28a) zu reinterpretieren.

(28) Brettsichere Surfer zieht es jedoch weiter in den Osten: Hier rauschen die Wogen des Atlantiks wesentlich rauer gegen die Küste als das Karibische Meer auf der anderen Seite.

(28a) Ostriel'aných surférov to však t’ahá/láka d’alej na východ: Tu vlny Atlantiku narážajú do brehu ovel'a drsnejšie ako vlny Karibského mora na druhej strane.

\subsubsection{Die direktionale Präposition in}

Die Korpusanalyse zeigt, dass die Subjektergänzungen, die mit der Direktionalpräposition in auftreten, mit dem Verb rauschen, in Bezug auf die Auffassung der Direktionalität, nicht-metaphorische (29) oder metaphorische (30) Syntagmen bilden. In Satz (29) geht es um eine schnelle Bewegung des Wassers, die aufgrund einer technischen Kraft entsteht, wobei diese Bewegung auch mit einem lauten Geräusch begleitet ist. In Satz (30) fehlt eine reale Bewegung, wie auch ein Geräusch, die metaphorische verbale Situation bezeichnet dank der abstrakten Subjektergänzung der Kurs und der Direktionalergänzung in den Keller das Sinken eines Finanzwertes. Die slowakische Entsprechung valit'sa in (29a) ist 
ein imperfektives Prädikat, die Entsprechung klesnút in (30a) ist hingegen ein perfektives Prädikat.

(29) Das Wasser rauschte dampfend in die Wanne.

(29a) Voda sa valila pariac do vane.

(29b) Voda sa valila do vane, pričom sa nad ňou dvíhala para.

(29c) Pariaca voda sa valila do vane.

(30) Als der Kurs in den Keller rauschte und die Banken die Kredite fällig stellten, waren die einst Wohlhabenden schnell überschuldet.

(30a) Ked' kurz klesol a banky zrušili úvery, kedysi bohatí l'udia sa rýchlo zadížili.

\section{Zusammenfassung}

Das Ziel des Beitrags lag darin, auf die Polysemie der Verben hinzuweisen, die auf der Schnittstelle der Syntax, Semantik und Pragmatik entstehen kann. Die Verbbedeutung ist in diesem Sinne ein komplexes Produkt, das durch den Sprachgebrauch selbst motiviert ist. Im Bereich der Valenzgrammatik heißt dies, dass das Valenzpotenzial eines Verbs innerhalb eines Satzes betrachtet werden muss.

Es wurde zu zeigen versucht, dass die Veränderung der lexikalischen Mittel, d.h. der lexikalische Übergang aus dem Bereich Geräusch in den Bereich Bewegung, nicht nur eine syntaktische, sondern auch eine semantische Veränderung bewirkt. Eine solche Veränderung veranschaulicht das Polysemieprinzip im Allgemeinen. Wenn eine lexikalische Einheit ein neues relevantes Sem erhält, wird die Bedeutung des jeweiligen Lexems modifiziert. Dadurch wird die Grammatikalisierung der Bedeutung abgeschwächt, und es öffnet sich der Raum zur Ausweitung des jeweiligen semantischen Feldes aus dem Bereich des Geräusches in den Bereich der Bewegung. Dies lässt sich an den direktionalen Adverbialien exemplifizieren, deren Vorkommen in einem Satz mit dem Geräuschverb rauschen nicht nur eine Valenzerweiterung, sondern auch eine Bedeutungsveränderung des Verbs bewirkt. Den resultativen Charakter der Konstruktionen mit den Direktionalien bestätigen auch perfektive Entsprechungen im Slowakischen.

Die Gegenüberstellung der deutschen Konstruktionen mit dem Verb rauschen im Kontrast zu deren Äquivalenten im Slowakischen macht deutlich, dass den deutschen analytischen resultativen Konstruktionen mit dem Verb rauschen die synthetischen perfektiven Prädikate entsprechen. Die nicht resultativen Konstruktionen, vor allem durch die prozessualen Adverbialien bedingt, ergeben im Slowakischen imperfektive Prädikate. 


\section{Literatur}

\section{Quellen}

Bechmann, Sascha (2013): Bedeutungswandel deutscher Verben. Eine gebrauchstheoretische Untersuchung. Tübingen.

Blank, Andreas (1997): Prinzipien des lexikalischen Bedeutungswandels am Beispiel der romanischen Sprachen. Tübingen.

Bußmann, Hadumod (1990): Lexikon der Sprachwissenschaft. Zweite, völlig neu bearbeitete Auflage. Stuttgart.

Connor, Kathleen / Kogan, Nathan (1980): Topic-Vehicle Relations in Metaphor: The Issue of Asymmetry. In: Honeck, Richard P. / Hoffman, Robert R. (Hrsg.): Cognition and Figurative Language. Hillsdale. S. 283-308.

Dölling, Johannes (1999): Formale Analyse von Metonymie und Metapher. In: Eckardt, Regine / von Heusinger, Klaus (Hrsg.): Meaning Change - Meaning Variation. Konstanz. S. 31-53.

Dvonč, Ladislav et al. (1966): Morfológia slovenského jazyka. Bratislava.

Engel, Ulrich (2004): Deutsche Grammatik. Neubearbeitung. München.

Engelberg, Stefan (2009): Blätter knistern über den Beton. Zwischenbericht aus einer korpuslinguistischen Studie zur Bewegungsinterpretation bei Geräuschverben. In: Winkler, Edeltraud (Hrsg.): Konstruktionelle Varianz bei Verben. Mannheim. S. 75-97.

Eroms, Hans-Werner (2012): Die Grenzen der Valenzen. In: Fischer, Klaus / Mollica, Fabio (Hrsg.): Valenz, Konstruktion und Deutsch als Fremdsprache. Frankfurt am Main u.a. S. 25-46.

Fleischer, Wolfgang / Barz, Irmhild (1995): Wortbildung der deutschen Gegenwartssprache. 2. durchgesehene und ergänzte Aufl. Tübingen.

Glück, Helmut (Hrsg.) (2005): Metzler Lexikon Sprache. Dritte, neubearbeitete Auflage. Stuttgart.

http://homepage.univie.ac.at/thomas.brooks/Welke_Valenz\%20und $\% 20$ Konstruktionletzt $\% 5 \mathrm{~B} 2 \% 5 \mathrm{D}$. pdf (Zugriff am 12.09.2016).

Keller, Rudi / Kirschbaum, Ilja (2003): Bedeutungswandel. Eine Einführung. Berlin.

Kozmová, Ružena (2004): Slovesný čas v nemeckom a slovenskom jazyku. Bratislava.

Kozmová, Ružena (2013): Quo vadis, Valenz? In: Bartoszewicz, Iwona / Hałub, Marek / Małyszek, Tomasz / Tomiczek, Eugeniusz (Hrsg.): Beobachtungen und Überlegungen. Wrocław. S. $115-130$.

Kozmová, Ružena (2013): Von der Funktion zur Bedeutung: Verbvalenz kontrastiv. Berlin.

Krupa, Viktor (1990): Metafora na rozhrani vedeckých disciplín. Bratislava.

Lohde, Michael (2006): Wortbildung des modernen Deutschen: ein Lehr- und Übungsbuch. Tübingen.

Ullmann, Stephen (1967/72): Grundzüge der Semantik. 2. unveränderte Auflage. Berlin.

Welke, Klaus (2005): Deutsche Syntax funktional. Perspektiviertheit syntaktischer Strukturen. 2. bearb. Auflage. Tübingen.

Welke, Klaus (2015): Wechselseitigkeit von Valenz und Konstruktion: Valenz als Grundvalenz. In: Engelberg, Stefan / Meliss, Meike / Proost, Kristel / Winkler, Edeltraud (Hrsg.): Argumentstrukturen zwischen Valenz und Konstruktion. Tübingen.

Germanica Wratislaviensia 143, 2018

(C) for this edition by CNS 


\section{Sekundärliteratur}

COSMAS II = Corpus Search, Management and Analysis System : www.ids-mannheim.de/cosmas2/ (Zugriff am 2.01.2017).

DWDS = Digitales Wörterbuch der deutschen Sprache: www.dwds.de/ (Zugriff am 2.01.2017).

Duden = Deutsches Universalwörterbuch: www.duden.de (Zugriff am 2.01.2017).

Kookkurrenzdatenbank CCDB von Cyril Belica: corpora.ids-mannheim.de/ccdb/ (Zugriff am 12.12.2016).

LEO Online-Wörterbuch: www.leo.org/ende/index_en.html (Zugriff am 12.12.2016).

PONS Online-Wörterbuch: de.pons.com/ (Zugriff am 12.12.2016).

Sketch Engine = language corpus management and query system: www.sketchengine.co.uk/ (Zugriff am 2.01.2017).

Slovensko-nemecký paralelný korpus: korpus.juls.savba.sk/skde.html (Zugriff am 2.01.2017).

The Free Dictionary: de.thefreedictionary.com/ (Zugriff am 12.12.2016).

Wörterbuch für Englisch-Deutsch und andere Sprachen: www.dict.cc/ (Zugriff am 12.12.2016).

Wörterbücher der Slowakischen Akademie für Wissenschaften: slovniky.juls.savba.sk/ (Zugriff am 12.12.2016). 